\title{
Quantized Peak Based Impulsive Noise Blanking in Power-Line Communications
}

\author{
Khaled M. Rabie, Student Member, IEEE, and Emad Alsusa, Senior Member, IEEE
}

\begin{abstract}
Impulsive noise (IN) over power-line channels can cause serious performance degradations. As such, many IN mitigation techniques have been proposed in the literature, the most common of which is the blanking technique. The conventional way to implement this technique however requires prior knowledge about the IN characteristics to identify the optimal blanking threshold (OBT). When such knowledge cannot be obtained the performance deteriorates rapidly. To alleviate this, we propose a look-up table (LUT) based algorithm with uniform quantization to utilize estimates of the peakto-average power ratio (PAPR) at the receiver to determine the OBT. To fully evaluate the performance of the proposed method we investigate the impact of quantization bits on the system performance in terms of signal-to-noise ratio (SNR) and symbol error rate under various IN scenarios. The results reveal that a 5 bit LUT is sufficient to achieve a gain of up to $3 \mathrm{~dB}$ SNR improvement relative to the conventional blanking method. It will also be shown that to maintain a good performance the resolution of quantization must be increased especially when the IN probability of occurrence is relatively high.
\end{abstract}

Index Terms-Blanking, impulsive noise, peak-to-average power ratio (PAPR), power-line communications (PLC), signal-to-noise ratio (SNR), symbol error rate (SER), uniform quantization.

\section{INTRODUCTION}

$\mathbf{P}$ OWER-LINE communications (PLC) technology is becoming a more attractive alternative for inhome networking applications competing with other technologies such as Ethernet and Wi-Fi. The main advantage of PLC is the fact that it exploits a pre-installed infrastructure of wiring networks. In addition, the availability of power outlets in every room allows easy access by the users. On the other hand, reliable communications over such channels require overcoming many challenges including noise [1]-[3], high levels of frequency-dependent attenuation [4] and multipath propagation [5]. Noise over power-line channels is generally categorized into background noise and impulsive noise (IN) [1], [6]; the latter is, however, the major factor responsible for degrading the performance of PLC systems [7]. IN has a short duration with random occurrence rate and a high power spectral density (PSD) [8] which is always $10-15 \mathrm{~dB}$ higher than the PSD of background noise and may reach as much as $50 \mathrm{~dB}$ [1]. In order to evaluate the system performance over IN channels, an accurate noise model is required. The most widely accepted analytical model is the Middleton class-A noise model [9], [10] which will be adopted in our investigations.

Many studies have been carried out on the topic of mitigating IN over power-line channels particularly for multicarrier modulation based systems such as OFDM which is associated with simple channel equalization methods. To overcome IN, the OFDM demodulator is preceded with a blanker to zero the incoming signal when it exceeds a certain threshold [11]-[14]. This method

The authors are with the School of Electrical and Electronic Engineering, the University of Manchester, Manchester, M13 9PL, UK. (khaled.rabie@manchester.ac.uk; e.alsusa@manchester.ac.uk). is widely used in practice because of its simplicity and ease of implementation [15], [16]. Theoretical performance analysis and optimization of blanking was first investigated by Zhidkov in [13], [17] where closed-form expressions for the signal-tonoise ratio (SNR) at the output of the blanker and the optimal blanking threshold (OBT) were derived. These studies rely on the assumption that the IN characteristics, in the form of signal-toimpulsive noise ratio (SINR) and the IN probability of occurrence, can be made available at the receiver in order to optimally blank the IN. This method is referred to here as the conventional optimal blanking (COB) method. Such assumptions constrain the applicability of this method and can be difficult to accomplish in practice. In [18], it is shown that even for small error estimations of the OBT, the COB method can suffer from significant performance degradation. Furthermore, the authors introduced a different criterion for estimating the OBT independently of the IN parameters by using estimates of the transmitted signals' peak to average power ratio (PAPR); this method was referred to as dynamic peak based threshold estimation (DPTE) method. The DPTE technique not only completely eliminates the need for prior knowledge about the characteristics of IN but can also achieve a gain of up to $2.5 \mathrm{~dB}$ if the signal peaks can be estimated accurately.

The question that arises here is, however, how can the signal peaks be determined for every single OFDM symbol at the receiver. In this paper, we propose and implement a technique to accomplish this by exploiting a look-up table (LUT) based algorithm with uniform quantization. This technique will be referred to as DPTE-LUT method. The OFDM symbol peaks are quantized and the corresponding bits are transmitted to the receiver as side information. The contribution of this paper resides in proposing a method for exploiting quantized estimates of the signal peak to estimate the OBT. Two aspects of the achievable performance were considered. The first is the impact of the LUT size on the different implementations of DPTE-LUT technique in terms of the output SNR and symbol error rate (SER) under various IN conditions. The second aspect is examining the effect of quantization concentration (window size). The results reveal that the proposed can provide up to $3 \mathrm{~dB}$ SNR enhancement in comparison with the $\mathrm{COB}$ method. It is also found that as the LUT size increases, the system performance improves, but more side information will be required at the receiver to identify the symbol peaks. Furthermore, it will be shown that the importance of quantization resolution becomes more significant than the number of quantization bits in a heavily disturbed IN environment. Under such conditions, it is found that for a LUT size of 2 bits, considering only $80 \%$ of the peaks spectrum yields almost $1 \mathrm{~dB}$ SNR improvement than including $99.5 \%$.

The remainder of this paper is organized as follows. In Section II the system model is described. In Section III the proposed 


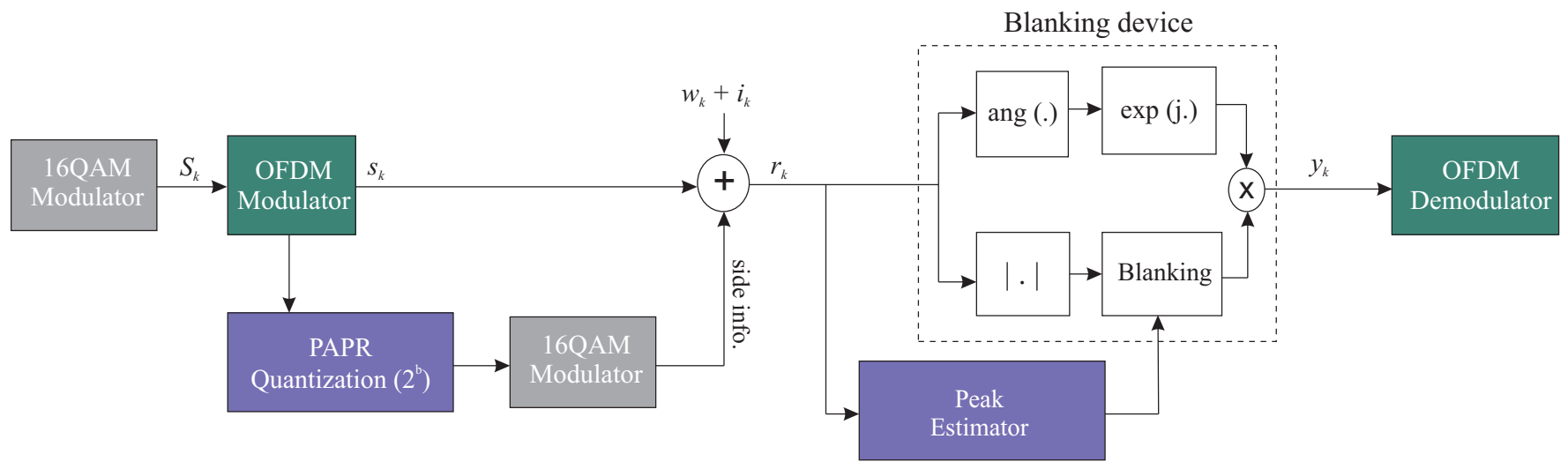

Figure 1: Block diagram of the proposed DPTE-LUT system

technique is demonstrated and a detailed discussion on OFDM symbol peak distribution is presented. Simulation results are presented in Section IV, including the effect of LUT size and the window size on the system performance. Finally, conclusions are drawn in Section V.

\section{System MOdEL}

Fig. 1 shows the basic block diagram of the system under consideration. The information bits are mapped into 16 quadrature amplitude modulation (16QAM) baseband symbols $S_{k}$. Then, the 16QAM signal is passed through an OFDM modulator to produce a time domain signal

$$
s(t)=\frac{1}{\sqrt{N}} \sum_{k=0}^{N-1} S_{k} e^{\frac{j 2 \pi k t}{T_{s}}}, \quad 0<t<T_{s}
$$

where $S_{k}$ is the complex constellations of the data symbols, $N$ is number of subcarriers and $T_{s}$ is the active symbol interval. The PAPR of the transmitted signal is expressed as

$$
\text { PAPR }=\frac{\max |s(t)|^{2}}{\mathbb{E}\left[|s(t)|^{2}\right]}, \quad 0<t<T_{s}
$$

where $\mathbb{E}[$.$] is the expectation function. Oversampling is usually$ employed when dealing with PAPR which can provide accurate estimates of the actual PAPR. Therefore, in this paper oversampling by 4 times is implemented as such oversampling rate was shown in the literature to provide a sufficiently accurate estimate. In this paper we deploy a special case of Middleton class-A noise model in which IN is characterized as a Bernoulli-Gaussian random process [19] and is given by

$$
n_{k}=w_{k}+i_{k}
$$

where

$$
i_{k}=b_{k} g_{k}, \quad k=0,1,2, \ldots, N-1
$$

$n_{k}$ is the total noise component, $w_{k}$ is the additive white Gaussian noise (AWGN), $i_{k}$ is the IN, $b_{k}$ is the Bernoulli process with probability $\operatorname{Pr}\left(b_{k}=1\right)=p$ and $g_{k}$ is complex white Gaussian noise with mean zero. The probability density function (PDF) of the total noise $n_{k}$ can be written as

$$
P_{n_{k}}\left(n_{k}\right)=(1-p) \mathcal{G}\left(n_{k}, 0, \sigma_{w}^{2}\right)+p \mathcal{G}\left(n_{k}, 0, \sigma_{w}^{2}+\sigma_{i}^{2}\right)
$$

where $\mathcal{G}($.$) is the Gaussian PDF, \sigma_{w}^{2}$ and $\sigma_{i}^{2}$ are the AWGN and IN variances, respectively. Under perfect synchronization condition, the received signal is given as [19]

$$
r_{k}=s_{k}+w_{k}+i_{k}, \quad k=0,1,2, \ldots, N-1
$$

$s_{k}=s\left(k T_{s} / N\right) ; s_{k}, w_{k}$ and $i_{k}$ are assumed to be mutually independent.

In the COB method, a blanker is applied before the OFDM demodulator and its basic principle is

$$
y_{k}=\left\{\begin{array}{ll}
r_{k}, & \left|r_{k}\right| \leq T \\
0, & \left|r_{k}\right|>T
\end{array} \quad k=0,1, \ldots, N-1\right.
$$

where $T$ is the blanking threshold, $r_{k}$ and $y_{k}$ are the input and output of the blanker, respectively. In [17], it is presented that $y_{k}=\alpha s_{k}+d_{k}$ where $\alpha$ is the appropriately selected scaling factor and $d_{k}$ is the cumulative noise term. This decomposition is justified by the application of Bussgang's theorem [20]. It is also shown that when $\alpha$ is chosen as $\alpha=(1 / 2) \mathbb{E}\left[\left|y_{k} s_{k}^{*}\right|^{2}\right]$, a closed-form expression for the output SNR of the COB method can be expressed as

$$
\mathrm{SNR}_{C O B}=\left(\frac{\mathbb{E}\left[\left|y_{k}\right|^{2}\right]}{2 \alpha^{2}}-1\right)^{-1}
$$

where $\mathbb{E}\left[\left|y_{k}\right|^{2}\right]$ and $\alpha$ are defined by (8) and (9), respectively. It was shown that this expression works well when the IN characteristics are accurately known a priori. However, in the DPTE technique [18], the OBT is obtained independently of IN characteristics. The blanker is applied at the receiver where the peak of each OFDM symbol is determined and adaptive blanking is employed accordingly as illustrated in Fig. 1 where its basic principle is

$$
y_{k}=\left\{\begin{array}{ll}
r_{k}, & \left|r_{k}\right| \leq \tilde{\mathrm{P}} \\
0, & \left|r_{k}\right|>\tilde{\mathrm{P}}
\end{array} \quad k=0,1, \ldots, N-1\right.
$$

$\tilde{\mathrm{P}}$ is the estimated OFDM symbol peak value which is obtained as presented in the next section. 
$\mathbb{E}\left[\left|y_{k}\right|^{2}\right]=2+2\left(1+\sigma_{w}^{2}+p \sigma_{i}^{2}\right)-(1-p)\left\{T^{2}+2\left(1+\sigma_{w}^{2}\right)\right\} e^{-\frac{T^{2}}{2\left(1+\sigma_{w}^{2}\right)}}-p\left\{T^{2}+2\left(1+\sigma_{w}^{2}+\sigma_{i}^{2}\right)\right\} e^{-\frac{T^{2}}{2\left(1+\sigma_{w}^{2}+\sigma_{i}^{2}\right)}}$

$$
\alpha=1-\left(1+\frac{T^{2}}{2\left(1+\sigma_{w}^{2}\right)}\right)(1-p) e^{-\frac{T^{2}}{2\left(1+\sigma_{w}^{2}\right)}}-\left(1+\frac{T^{2}}{2\left(1+\sigma_{w}^{2}+\sigma_{i}^{2}\right)}\right) p e^{-\frac{T^{2}}{2\left(1+\sigma_{w}^{2}+\sigma_{i}^{2}\right)}}
$$

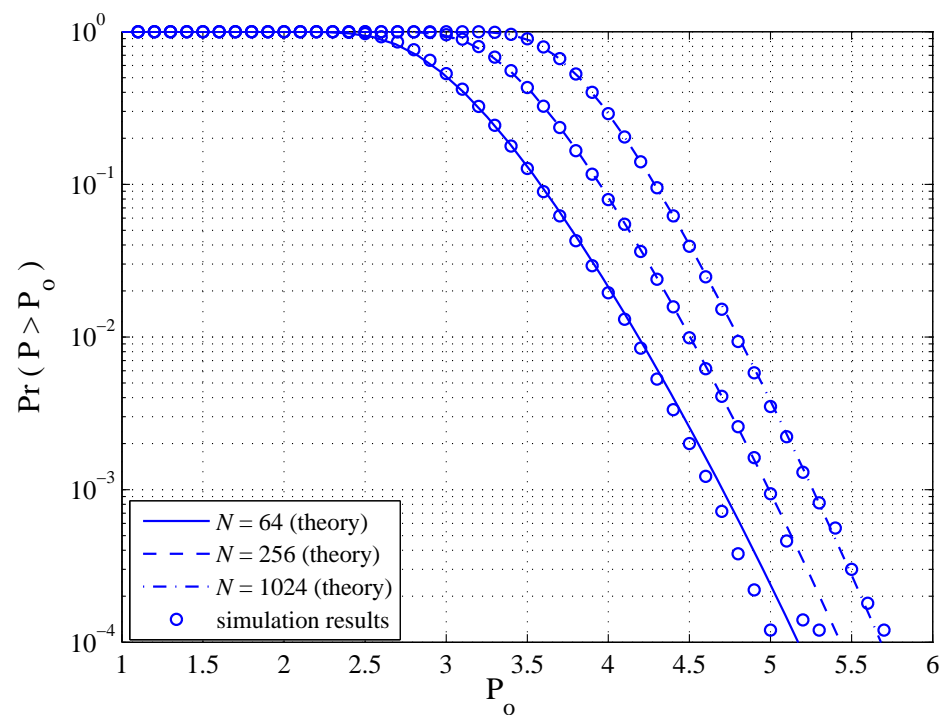

Fig. 2: CCDF plot for 16QAM OFDM system, $N=64,256$ and 1024

\section{The Proposed Method}

In this section the proposed method is described. For better realization of this method, it is important to analyze the peaks distribution of the OFDM signal. Therefore, we begin by introducing the complementary cumulative distribution function (CCDF) of the PAPR of the OFDM signal with $N$ subcarriers. The CCDF denotes the probability that the PAPR of a data block exceeds a given threshold $\left(\mathrm{PAPR}_{o}\right)$. This paper adopts the theoretical CCDF expression, derived in [21]. This expression can be rewritten in terms of peaks, $\mathrm{P}=\max |s(t)|$, instead of PAPR as

$$
\mathrm{CCDF}=1-\operatorname{Pr}\left(\mathrm{P} \leq \mathrm{P}_{o}\right)=1-\left(1-e^{\left(-\mathrm{P}_{\mathrm{o}}\right)}\right)^{N}
$$

It is worthwhile mentioning the fact that a more accurate expression for the CCDF of PAPR can be found in [22]. A plot of (12) along with simulation results are shown in Fig. 2 for OFDM signals with 64,256 and 1024 subcarriers. It is clear that both the analytical and simulation results are in good agreement. It is also noticeable that for $N=64$, the vast majority of the symbols have peaks between 2.5 and 3.5. For further clarity a bar-chart for the signal peaks distribution is plotted in Fig. 3. This figure provides useful information for instance, it can be seen that $99.5 \%$ of the symbol peaks, i.e. window size (WS $=99.5 \%$ ), are concentrated within the range from 2.2 to 4.3 whereas $80 \%$ of the peaks lie in the range between 2.6 and 3.4. Such information is advantageous for determining the best trade-off between the number of quantization bits, hence the size of the LUT, and WS.

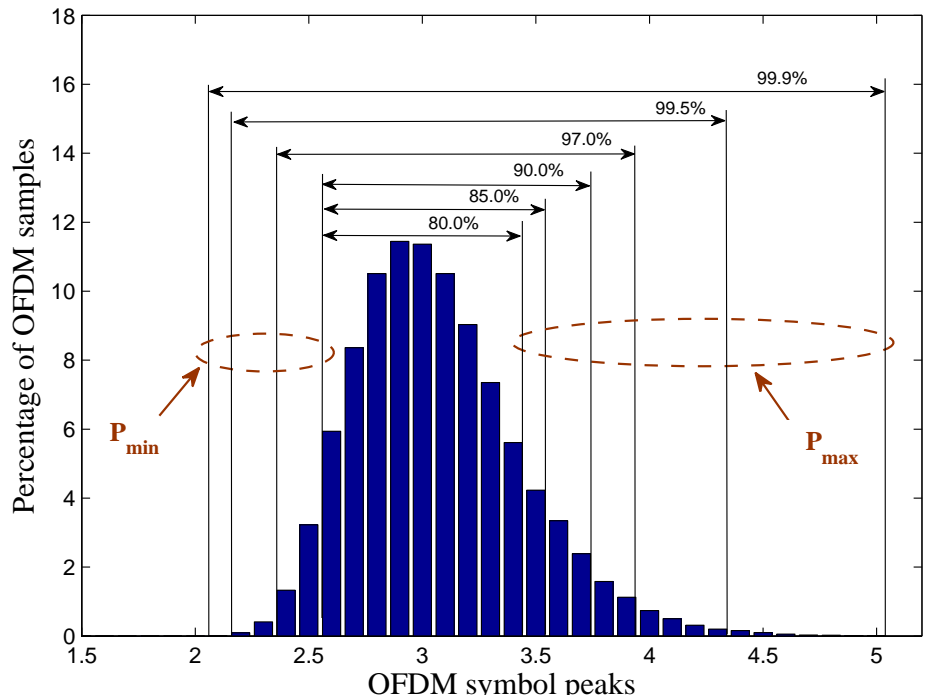

Fig. 3: OFDM symbol peak distribution

As mentioned earlier, the proposed technique utilizes a LUT, the size of which depends on the required accuracy of the signal peak estimate at the receiver. The symbol peak amplitudes can take on any value on a continuous range following the probabilistic model shown in Fig. 2 and therefore must be discretized into a finite number of quantized levels $\left(\mathrm{P}_{q}\right)$, where $q=\left\{1,2, \ldots, N_{q}\right\}$, ranging from predetermined minimum and maximum values $\mathrm{P}_{\text {min }}=\mathrm{P}_{1}$ and $\mathrm{P}_{\max }=\mathrm{P}_{N_{q}}$, respectively, see Fig. 3. $N_{q}$ depends on the size of the LUT being used $\left(N_{q}=2^{b}\right)$, where $b$ is the number of bits representing each OFDM symbol peak. Since the proposed technique exploits uniform quantization the resolution factor $\left(\mathcal{R}_{\mathcal{F}}\right)$, i.e. the spacing between quantization levels, can be defined as

$$
\mathcal{R}_{\mathcal{F}}=\frac{\mathrm{P}_{\max }-\mathrm{P}_{\min }}{N_{q}}
$$

The smaller the $\mathcal{R}_{\mathcal{F}}$, the better the precision of the signal peak estimates achieved. It is important to ensure that signal peaks which fall between two quantization levels are assigned to the upper level. This minimizes the possibility that the receiver will not blank the useful signal energy for that specific symbols. However, symbols with peaks larger than $\mathrm{P}_{\max }$ are mapped into $\mathrm{P}_{\max }$ and similarly all symbols having peaks below $\mathrm{P}_{\min }$ will be mapped into $\mathrm{P}_{\min }$. The quantized peaks are represented by $b$ bits per OFDM symbol which are transmitted to the receiver as side information using 16QAM modulation along with the useful data. However, the side information can also be transmitted over 


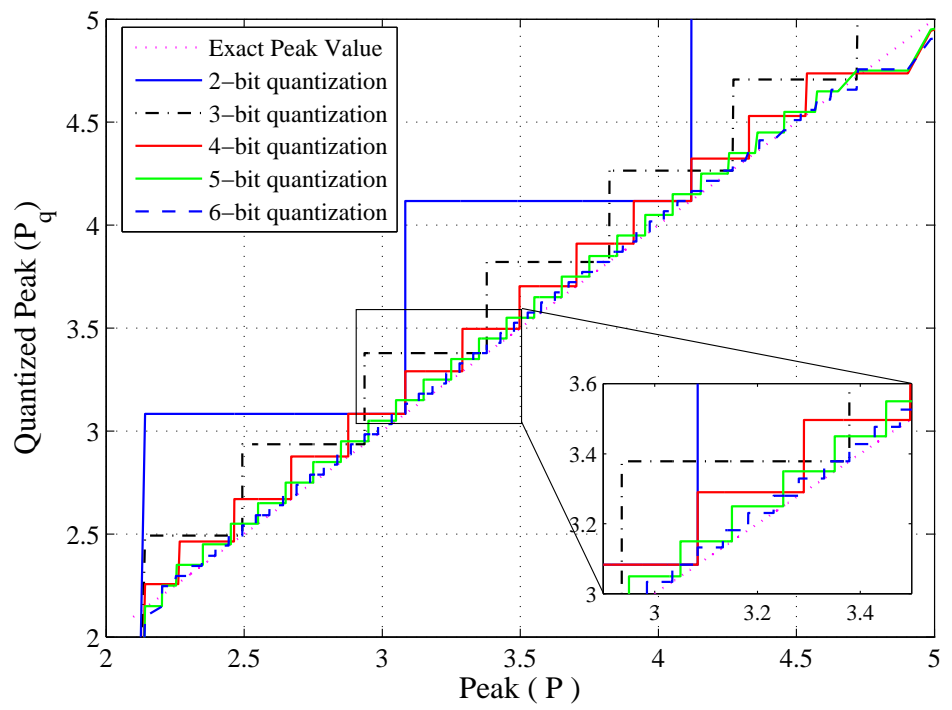

Fig. 4: Uniform quantization of OFDM symbol peaks

a separate control channel which can either occupy a different frequency band or utilize specific time slots. At the receiver, the peak estimator, shown in Fig. 1, will extract the peak value of the associated symbol and adjust the blanking threshold of the blanking device accordingly. The basic principle of the peak estimator can be divided into two operations. 1) The quantization bits of the associated symbol are extracted from the side information by performing a 16QAM demodulation process. 2) These bits are then utilized to find the corresponding symbol peak value $(\tilde{\mathrm{P}})$ from the predetermined LUT at the receiver. Fig. 4 shows the exact $(\mathrm{P})$ and quantized $\left(\mathrm{P}_{q}\right)$ signal peaks and it is clear that as the LUT size increases, the resolution becomes higher and consequently the quantization error $\left(e_{q}=\mathrm{P}-\mathrm{P}_{q}\right)$ is minimized as demonstrated in Fig. 5. This implies that more accurate estimation of the signal peaks can be obtained at the receiver, hence more accurate blanking threshold is used resulting in more efficient IN suppression.

\section{Simulation Results}

This section investigates the effect of the LUT size as well as the impact of quantization concentration (window size) on the DPTE-LUT method in terms of output SNR and SER under various IN scenarios. Our simulations are based on an OFDM system consisting of $N=64$ subcarriers with 16QAM modulation. It is assumed that the transmitter and receiver are synchronized and that the OFDM signal power is normalized to unity $\sigma_{s}^{2}=$ $(1 / 2) \mathbb{E}\left[\left|s_{k}\right|^{2}\right]=1, \sigma_{w}^{2}=(1 / 2) \mathbb{E}\left[\left|w_{k}\right|^{2}\right]$ and $\sigma_{i}^{2}=(1 / 2) \mathbb{E}\left[\left|i_{k}\right|^{2}\right]$. The input SNR and SINR are defined by

$$
\begin{aligned}
& \mathrm{SNR}=10 \log _{10}\left(\frac{1}{\sigma_{w}^{2}}\right) \\
& \mathrm{SINR}=10 \log _{10}\left(\frac{1}{\sigma_{i}^{2}}\right)
\end{aligned}
$$

For all the simulation results in this section, $\mathrm{SNR}=40 \mathrm{~dB}$ and the output SNR is determined as

$$
\mathrm{SNR}_{D P T E}=\frac{\mathbb{E}\left[\left|\alpha s_{k}\right|^{2}\right]}{\mathbb{E}\left[\left|y_{k}-\alpha s_{k}\right|^{2}\right]}
$$

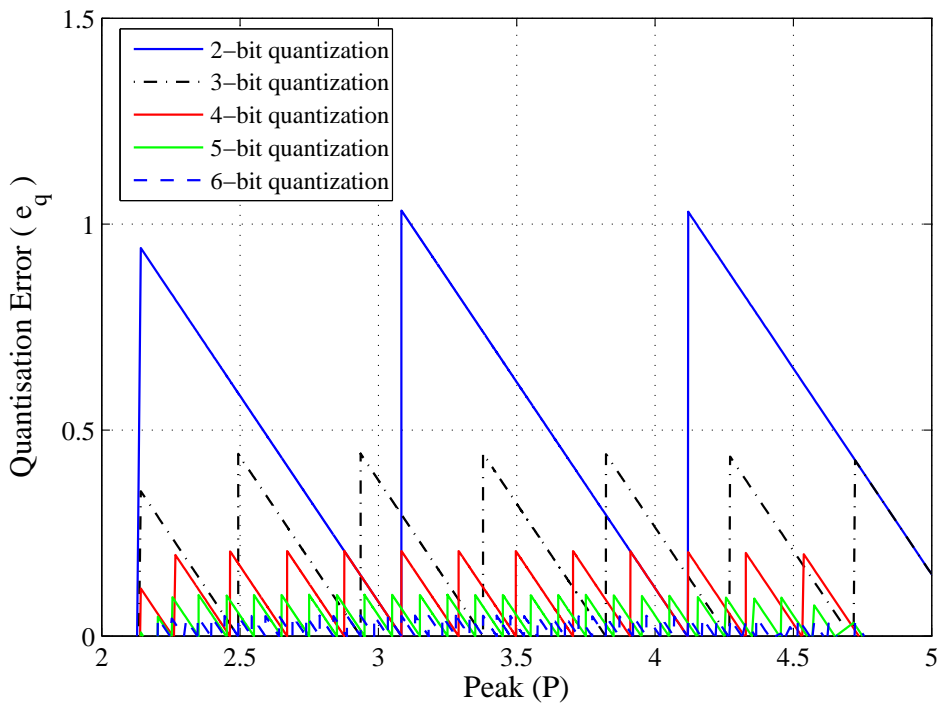

Fig. 5: Quantization error of OFDM symbol peaks

where $\alpha=(1 / 2) \mathbb{E}\left[\left|y_{k} s_{k}^{*}\right|^{2}\right]$. For better clarity it is important to define in advance the different DPTE scenarios considered here.

- Ideal DPTE: assumes exact signal peaks are determined precisely at the receiver. This establishes the lower bound performance of DPTE method.

- Ideal DPTE-LUT: means that the quantized signal peaks are detected at the receiver error-free, i.e. assuming that the side information is not contaminated with noise.

- Practical DPTE-LUT: this is the case when side information is passed through the PLC channel and experience IN impairments.

\section{A. The Effect of LUT Size}

In this subsection we examine the impact of the LUT size on the performance of both the ideal and practical DPTE-LUT techniques. In addition, the gain in the output SNR relative to the $\mathrm{COB}$ method is presented. This is referred to as the relative gain $\left(G_{R}\right)$ and is expressed as

$$
G_{R}=10 \log _{10}\left(\frac{\mathrm{SNR}_{D P T E}}{\mathrm{SNR}_{C O B}}\right)
$$

The results for the DPTE-LUT system, in this subsection, are obtained for a WS of $99.9 \%, \mathrm{P}_{\min }=2$ and $\mathrm{P}_{\max }=5$, see Fig. 3. The reason why a WS of $100 \%$ is not used is because such assumption will require $\mathrm{P}_{\max }=\infty$ which is not practical. Not only that, but also using a very large value of $\mathrm{P}_{\max }$ will lead to significant quantization error resulting in inefficient IN blanking.

1) The Ideal DPTE-LUT Technique: The output SNRs versus SINR for the COB, ideal DPTE and ideal DPTE-LUT techniques are shown in Fig. 6 for LUT sizes $\{b=2,3,4,5$ bits $\}$ and IN probabilities $\{p=0.001,0.01,0.1\}$. The results of the COB method are obtained using (10) under the assumption of perfect IN parameters estimation. As anticipated it can be seen from these results that as the LUT size increases, the performance of the DPTE-LUT scheme becomes closer to that of the ideal 


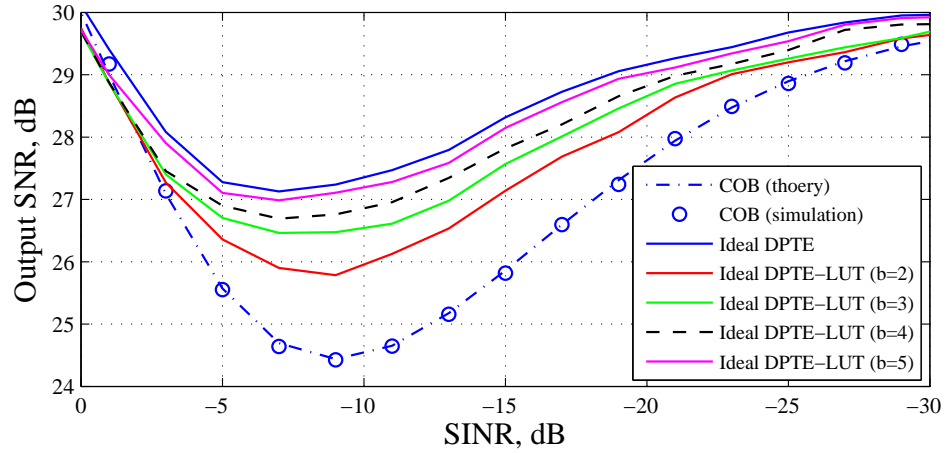

(a) $p=0.001$

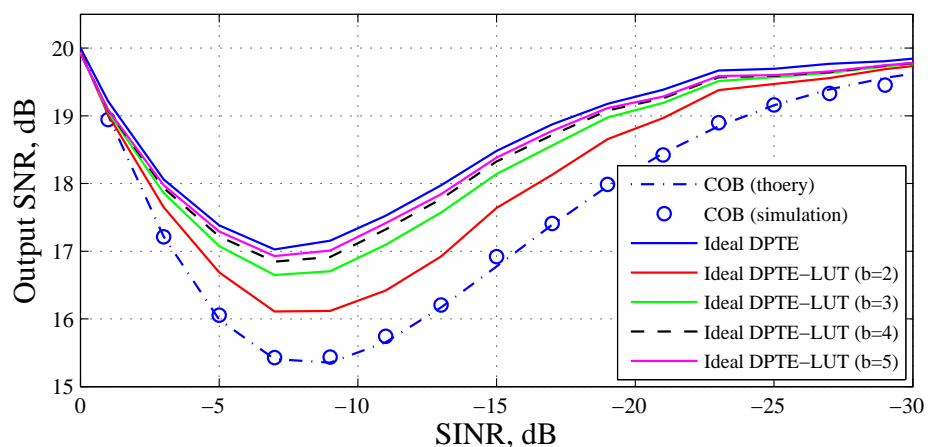

(b) $p=0.01$

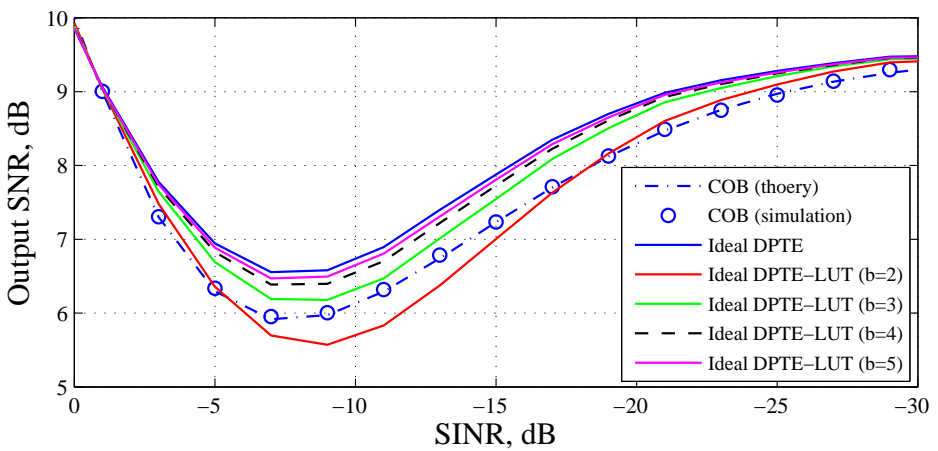

(c) $p=0.1$

Fig. 6: Output SNR of the COB, ideal DPTE and ideal DPTE-LUT methods versus SINR for various values of $p$

DPTE system. It is also evident that for low IN probabilities $\{p=0.001,0.01\}$, Fig. $6 \mathrm{a}$ and Fig. $6 \mathrm{~b}$, the proposed technique always outperforms the COB method irrespective of the LUT size. On the other hand, however, for heavily-disturbed IN channel $\{p=0.1\}$, Fig. 6c, the importance of LUT size becomes more significant. It is noticeable that when a LUT size of only 2 bits is used, the proposed scheme slightly under-performs the COB method in the intermediate SINR region $(-5 \mathrm{~dB} \rightarrow-15 \mathrm{~dB})$. This clearly states that higher resolution is required when the IN probability of occurrence is relatively high. This will be investigated thoroughly in the next subsection. In addition, it is worth pointing out that for all IN scenarios a LUT of size 4 or 5 bits is sufficient to achieve a near-ideal performance. It is obvious that for the ideal DPTE system, a gain of up to $2.75 \mathrm{~dB}, 1.75 \mathrm{~dB}$ and $0.6 \mathrm{~dB}$ can be attained when $p=0.001,0.01$ and 0.1 , respectively. This represents the highest achievable gain and it is clear that the proposed technique approaches this performance with a LUT of

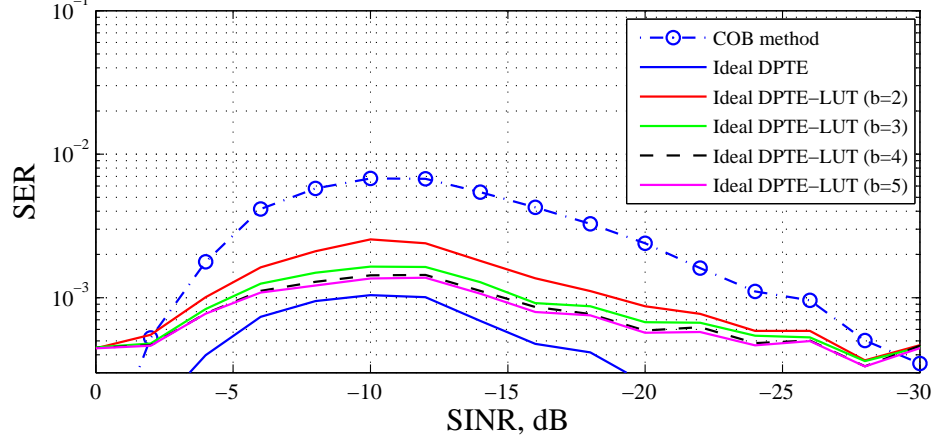

(a) $p=0.001$

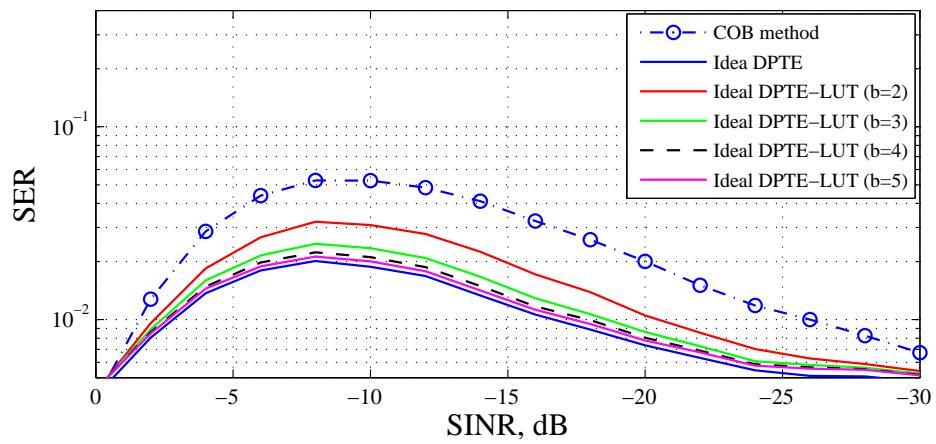

(b) $p=0.01$

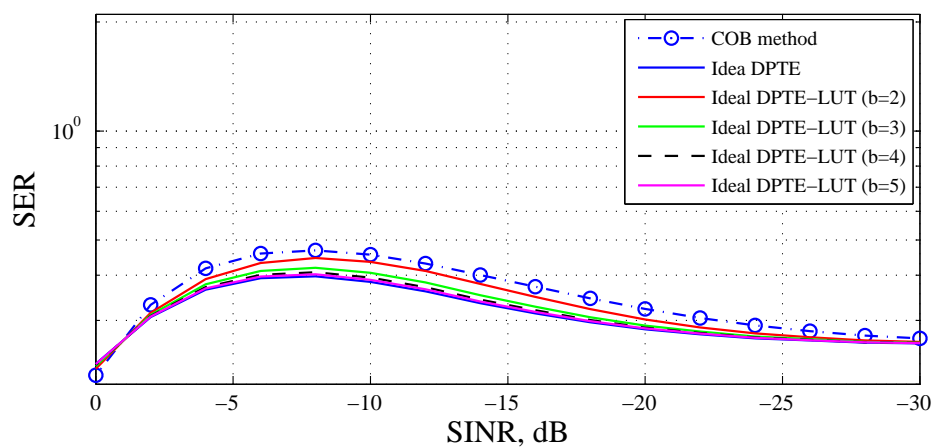

(c) $p=0.1$

Fig. 7: SER performance of COB, ideal DPTE and ideal DPTE-LUT methods versus SINR for various values of $p$

size 4 or 5 bits. Furthermore, the SER performance corresponding to the SNR results in Fig. 6 is presented in Fig. 7 and same trends can be observed.

2) The Practical DPTE-LUT Technique: The realization of the proposed scheme requires transmitting the side information associated with each OFDM symbol peak. In practice, however, the PLC channel is contaminated with noise which may lead to receiving some of such information in error. In this section we investigate the impact of practical implementation on the proposed system. Our investigations here will adopt a 4-bit LUT as such LUT size is found in the previous section to provide sufficiently accurate peak estimation. Fig. 8 compares the output SNRs for the ideal DPTE, ideal DPTE-LUT and practical DPTELUT techniques in addition to the $\mathrm{COB}$ method for various values of $p$. As expected, it is observed that the performance of the practical DPTE-LUT technique becomes closer to that of the ideal DPTE-LUT case as $p$ becomes smaller. This can be 


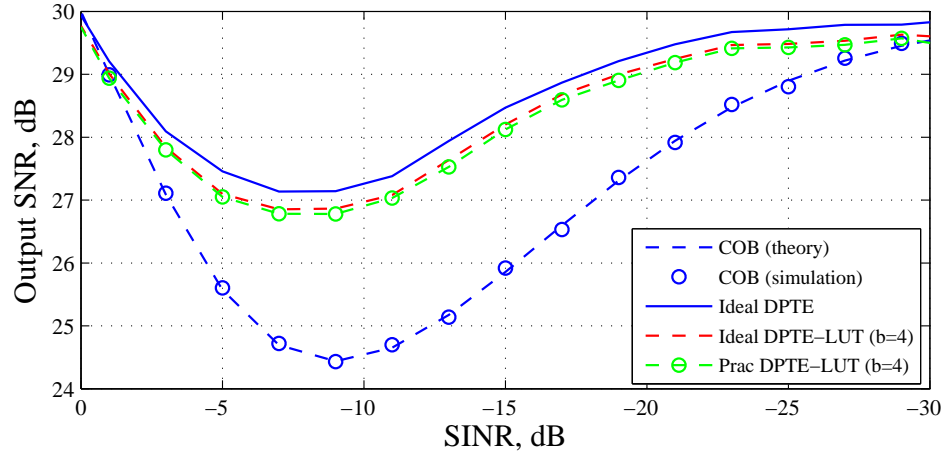

(a) $p=0.001$

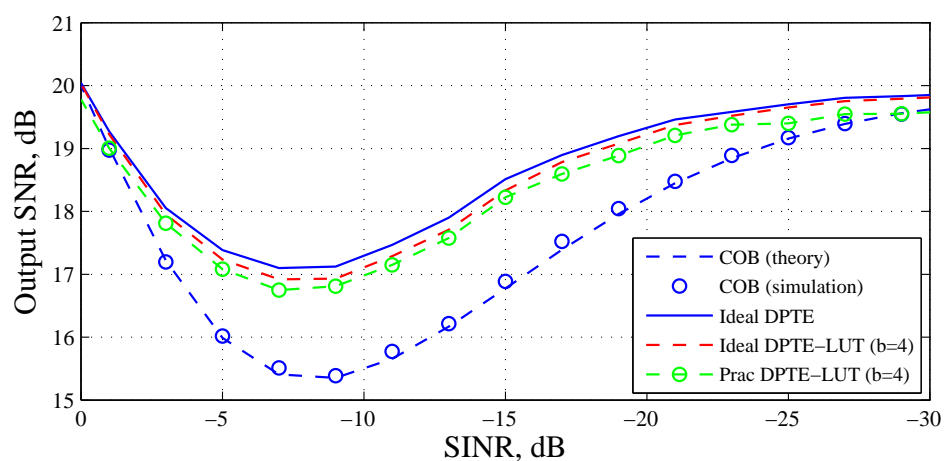

(b) $p=0.01$

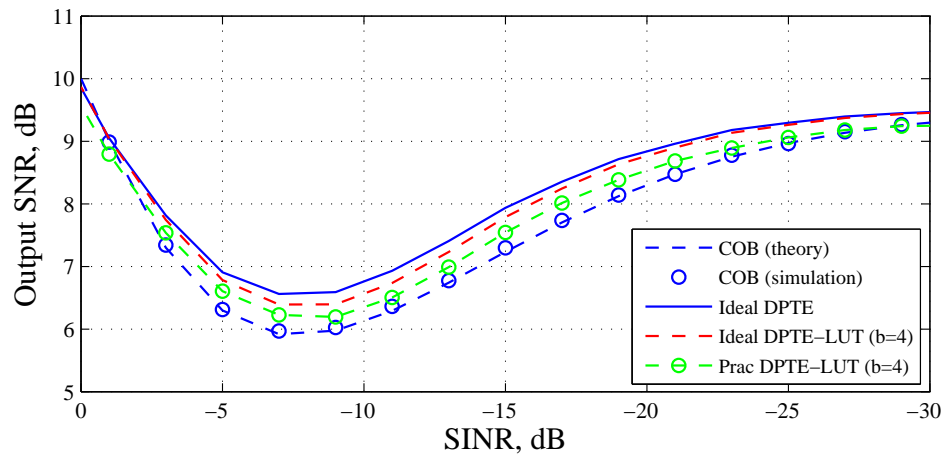

(c) $p=0.1$

Fig. 8: Output SNR of the COB, ideal DPTE, ideal DPTE-LUT and practical DPTE-LUT methods versus SINR for various values of $p$

justified as follows: when $p$ is high, the side information is more likely to be detected in error resulting in using the inaccurate blanking threshold and therefore, causing inefficient IN reduction. Furthermore, it is clearly seen that the loss due to the practical impact of IN on the side information is insignificant. Hence it can be concluded that the proposed technique is promising and can be reliably implemented in practice.

Similarly as in the previous section, the SER performance in correspondence to the SNR curves in Fig. 8 is depicted in Fig. 9 and similar observations can be seen. However, it is worthwhile stressing fact that the robustness of the proposed scheme can be further enhanced by applying powerful coding techniques to make the side information more resistant to IN. Moreover, it is important to mention that the impact of the number of subcarriers, for a given LUT size, on the proposed technique will be insignificant if $\mathrm{P}_{\min }$ and $\mathrm{P}_{\max }$ are chosen appropriately. This can be extracted

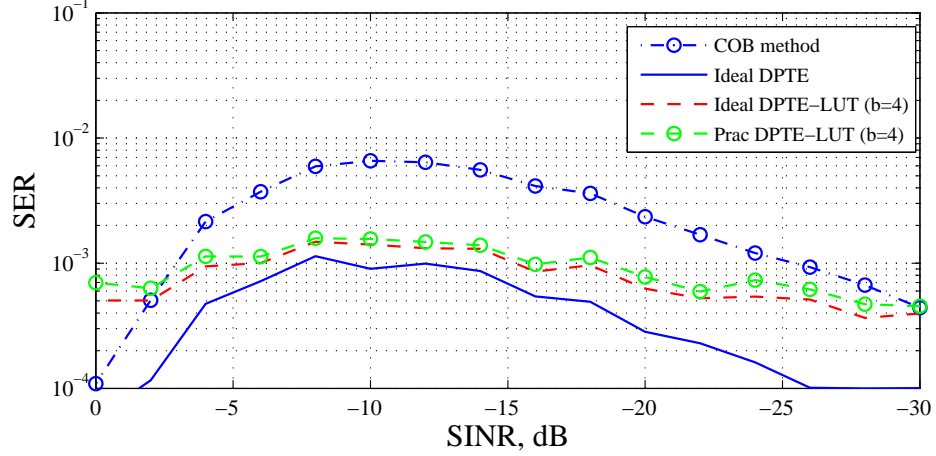

(a) $p=0.001$

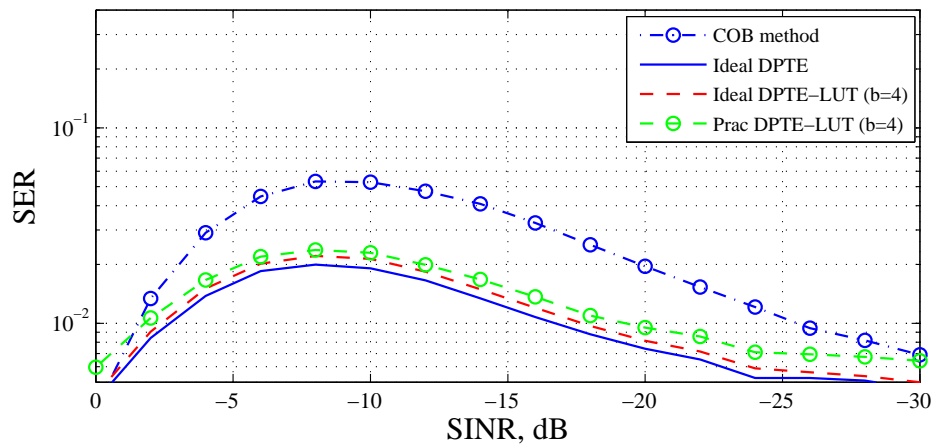

(b) $p=0.01$

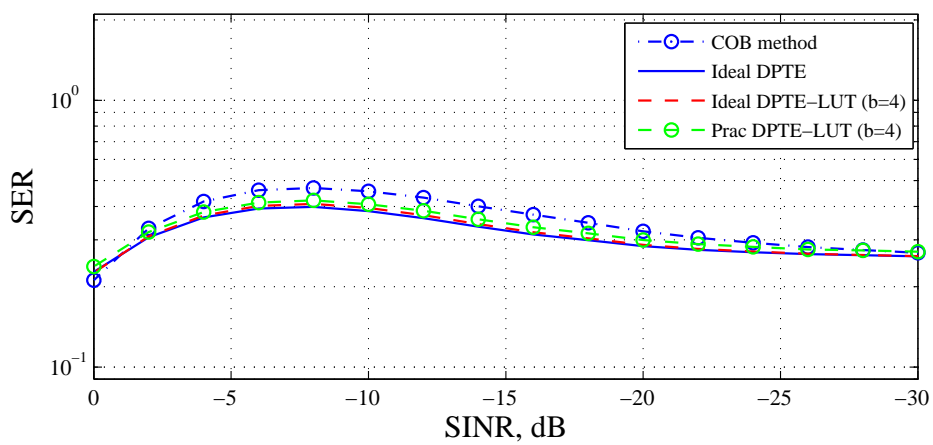

(c) $p=0.1$

Fig. 9: SER performance of the COB, ideal DPTE, ideal DPTE-LUT and practical DPTE-LUT methods versus SINR for various values of $p$

from the behavior of CCDF curves in Fig. 2 where the curves are identical but shifted. The shift to the right as $N$ increases implies that $\mathrm{P}_{\min }$ and $\mathrm{P}_{\max }$ should be increased for larger $N$ in order to obtain same precision.

\section{B. The Effect of Window Size}

In this subsection the concentration effect of quantization on the ideal DPTE-LUT scheme is assessed in two different environments, weakly and heavily disturbed IN. We define the relative loss $\left(L_{R}\right)$ as the loss in the output SNR of the proposed technique caused due to considering only part of the OFDM peaks spectrum and is given as

$$
L_{R}=10 \log _{10}\left(\frac{\mathrm{SNR}_{D P T E-L U T}}{\mathrm{SNR}_{D P T E-\text { Ideal }}}\right)
$$




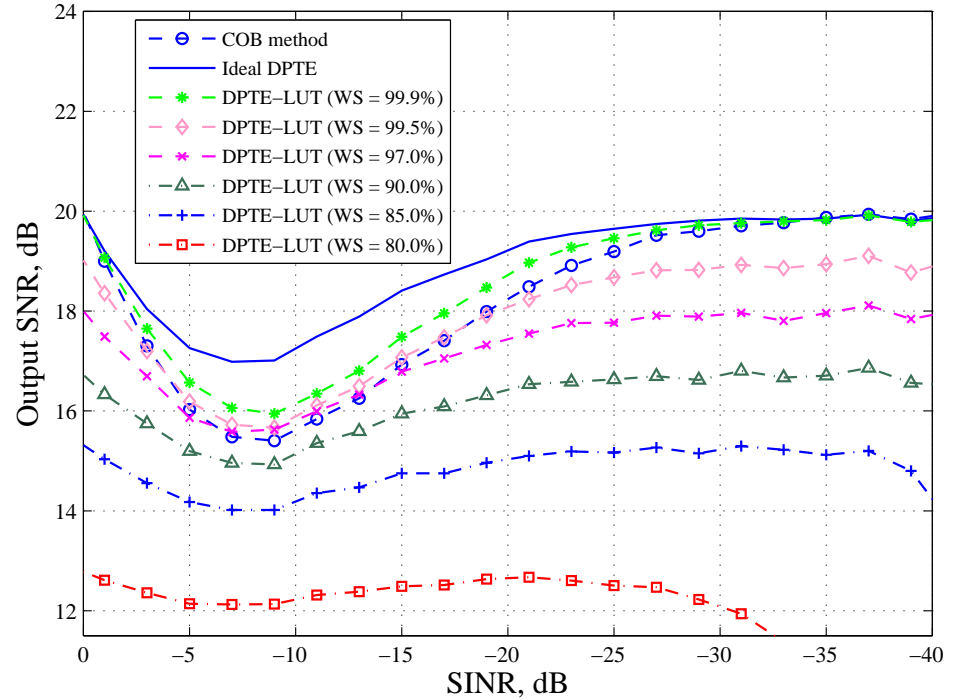

(a) $\mathrm{LUT}=2$ bits

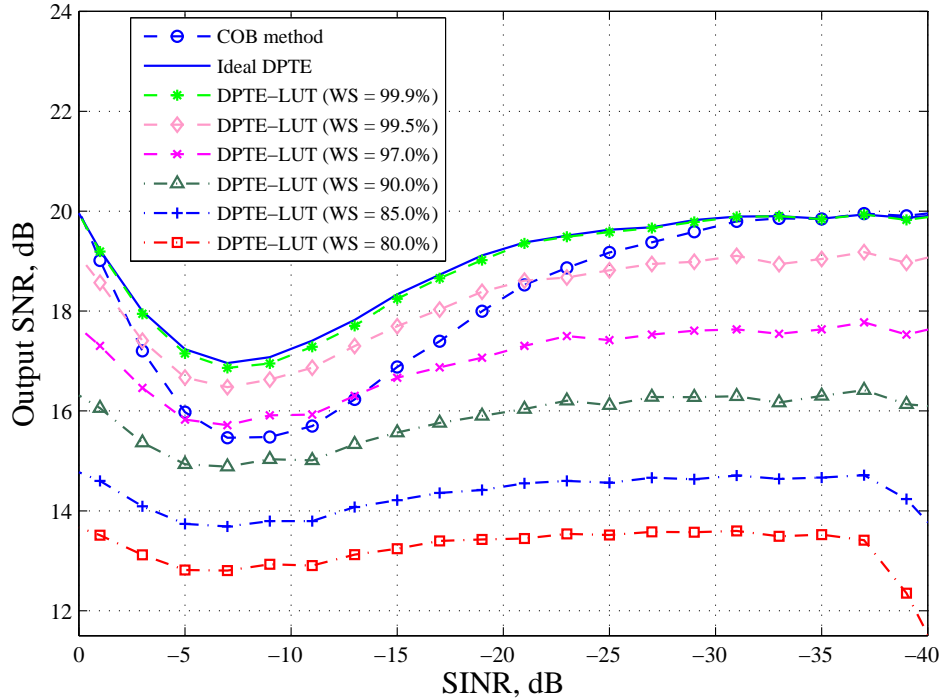

(b) LUT $=5$ bits

Fig. 10: Output SNR versus SINR for LUT $=2,5$ bits in a weakly-disturbed IN environment for different WS values

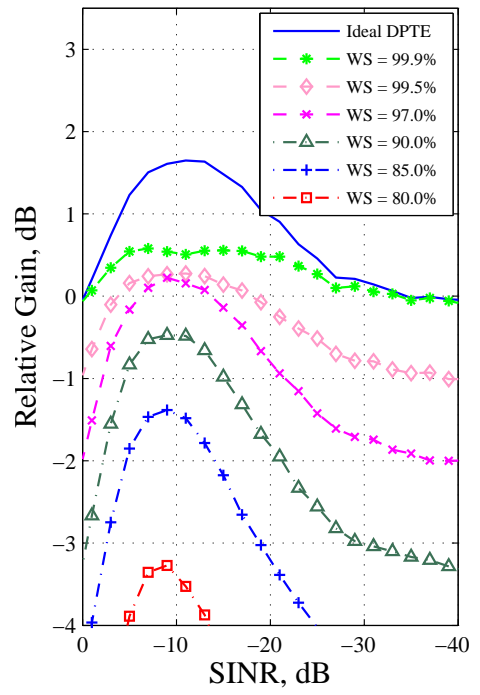

(a) LUT $=2$ bits

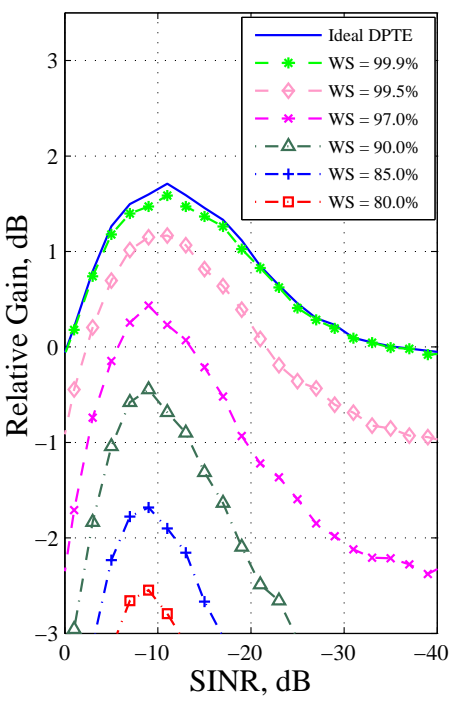

(b) LUT $=5$ bits
Fig. 11: Relative gain versus SINR for $\mathrm{LUT}=2,5$ bits in a weakly-disturbed IN environment for different WS values

1) Weakly Disturbed IN Environment : The output SNR of the proposed technique for different WSs when $\{p=0.01\}$ is illustrated in Fig. 10 for two LUT sizes $\{b=2,5$ bits $\}$. To obtain comparative figures, the SNR for the COB and the ideal DPTE techniques are also included on this plot. As can be seen, there is a general trend that as the WS increases, the proposed system performance becomes closer to that of the ideal DPTE scheme. This phenomenon is clearly highlighted in Fig. 11 which shows a plot of the $G_{R}$, given by (17). Moreover, the relative loss is illustrated in Fig. 12 and it is obvious that the loss increases considerably as WS becomes smaller. In general, it is also noted that for the same WS, the loss is smaller for a system with a LUT of 5 bits in relation to that with a LUT of only 2 bits.

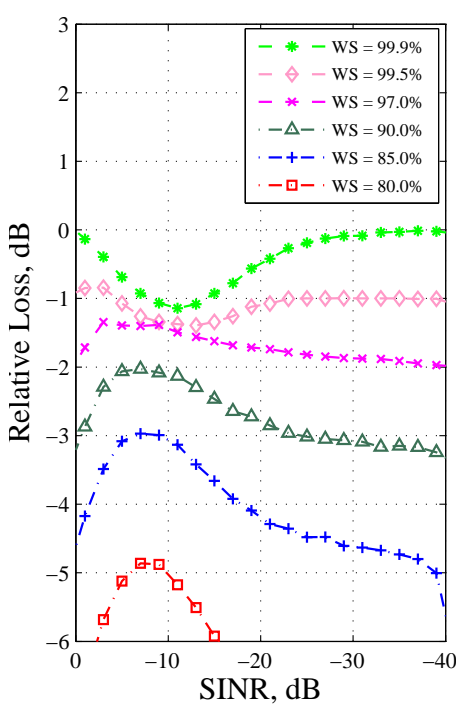

(a) $\mathrm{LUT}=2$ bits

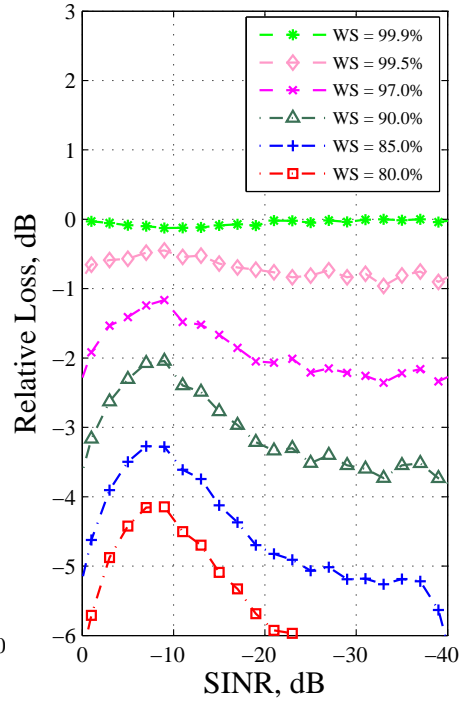

(b) LUT $=5$ bits
Fig. 12: Relative loss versus SINR for $\mathrm{LUT}=2,5$ bits in a weakly-disturbed IN environment for different WS values

2) Heavily Disturbed IN Environment: Similar procedure as in the previous section is followed here, however, for a heavilydisturbed IN environment $\{p=0.2\}$. Results of the output SNR, $G_{R}$ and $L_{R}$ are demonstrated in Figs. 13-15, respectively. It is interesting to note that for small LUT size, Figs. 13a, 14a and $15 \mathrm{a}$, in the intermediate SINR region, $\mathcal{R}_{\mathcal{F}}$ becomes more crucial to the overall performance than WS. For instance, we can see that considering only $80.0 \%$ of the peaks spectrum, in the intermediate range of SINR, yields up to $1 \mathrm{~dB}$ better SNR performance than that of wider WS such as $99.5 \%$ and $99.9 \%$. The intuitive explanation for this is the fact that in the intermediate SINR region the amplitude of IN values are slightly higher than the signal values which makes this region very sensitive and consequently any small quantization error in the peak values will result in a severe effect 


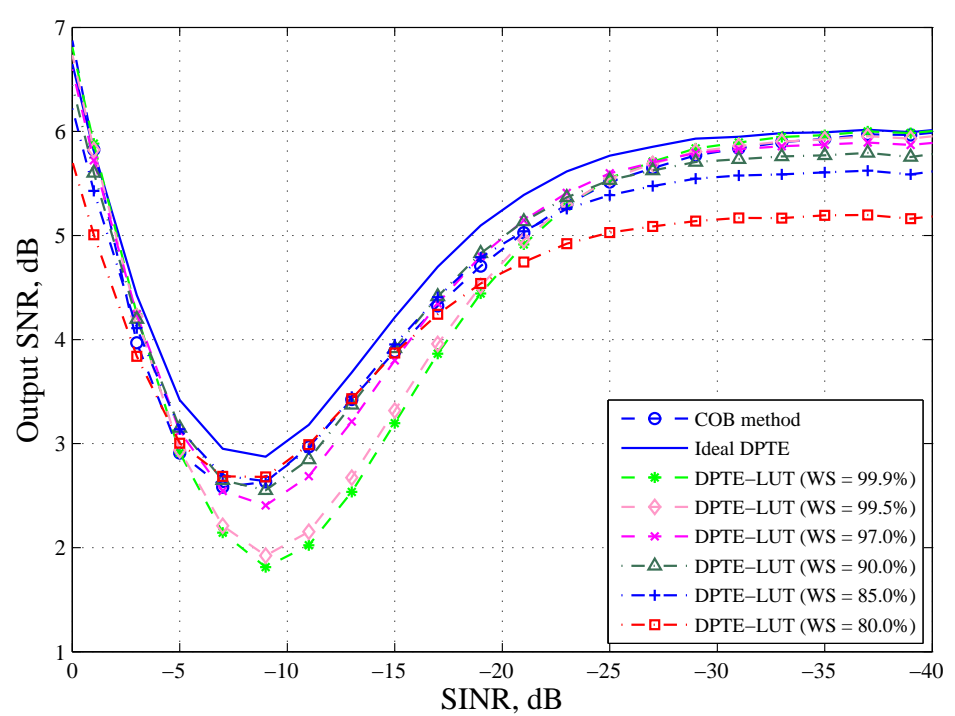

(a) LUT $=2$ bits

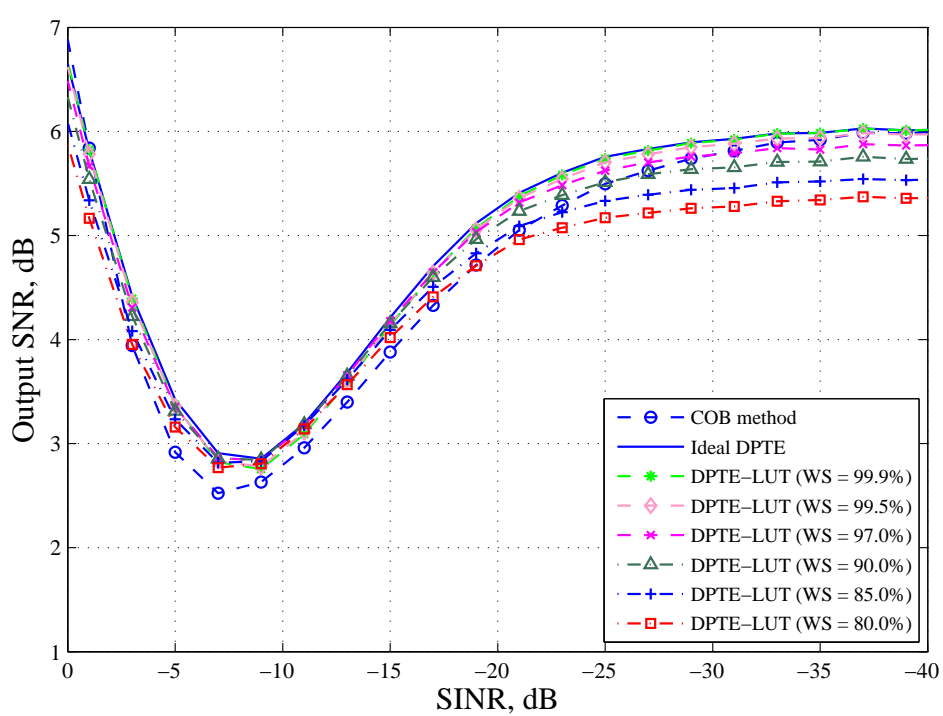

(b) LUT $=5$ bits

Figure 13: Output SNR versus SINR for LUT $=2,5$ bits in a heavily-disturbed IN environment for different WS values

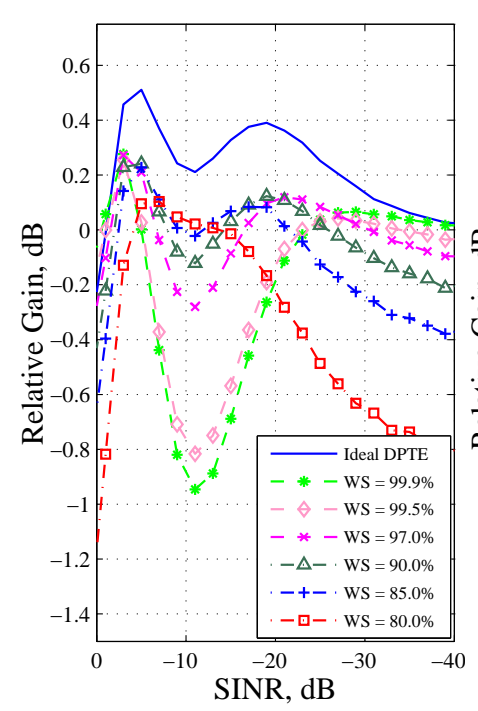

(a) $\mathrm{LUT}=2$ bits

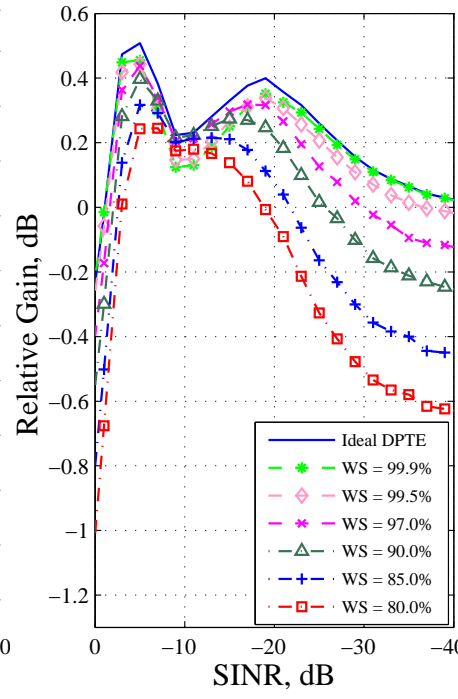

(b) LUT $=5$ bits
Figure 14: Relative gain versus SINR for $\mathrm{LUT}=2,5$ bits in a heavilydisturbed IN environment for different WS values

on the output SNR. In contrast, when LUT size is relatively big $\{b=5$ bits $\}$, Figs. $13 \mathrm{~b}, 14 \mathrm{~b}$ and $15 \mathrm{~b}$, the performance is almost independent of the WS since quantization resolution becomes sufficiently high for all WSs. Therefore, in such environments, the application of non-uniform quantization seems appropriate as quantization intervals can be made smaller where the majority of peaks are concentrated; hence, higher quantization accuracy can be achieved.

\section{CONCLUSION}

The effect of IN over power-lines can be dramatically minimized by applying a blanker at the receiver. Two different techniques can be used to implement this scheme, namely, COB and DPTE. The ideal DPTE technique establishes the lower bound

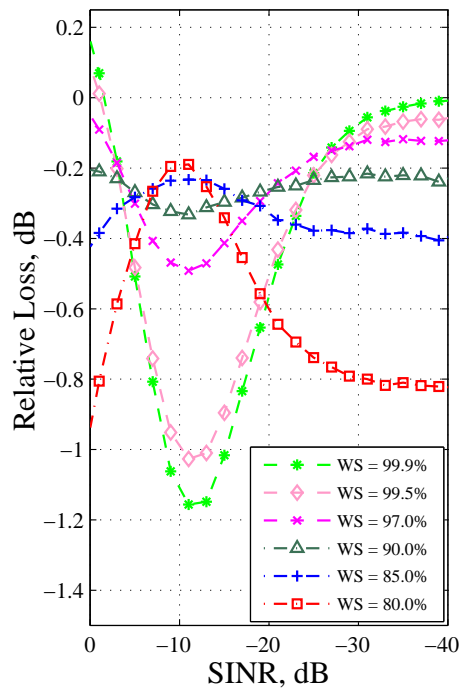

(a) $\mathrm{LUT}=2$ bits

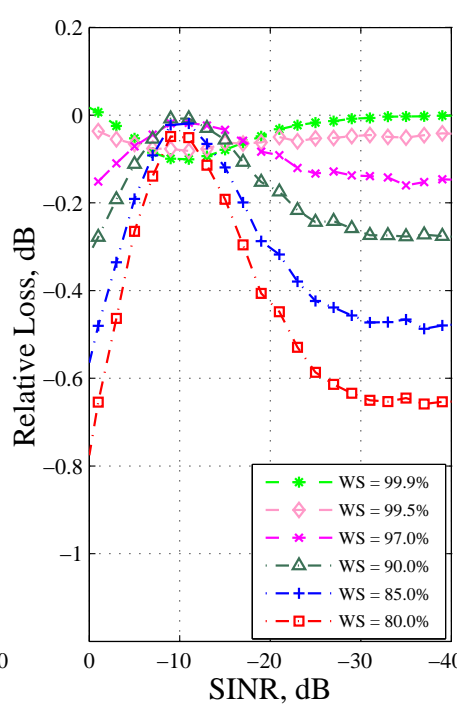

(b) $\mathrm{LUT}=5$ bits
Figure 15: Relative loss versus SINR for LUT $=2,5$ bits in a heavilydisturbed IN environment for different WS values

performance of this scheme which assumes that the peak of each OFDM symbol is known accurately at the receiver. In this paper we introduced a novel technique for estimating the signal peak and utilized it to reduce the effect of IN. This technique deploys a LUT based algorithm with uniform quantization and allows optimal blanking without the need to any IN measurements. It is found that as the LUT size increases, better performance is achieved. However it was demonstrated that, in general, a LUT size of 32 is sufficient to achieve near-ideal performance. It was also presented that in heavily-disturbed IN environment, more quantization accuracy is required, specially in the intermediate SINR region. 


\section{REFERENCES}

[1] M. Zimmermann and K. Dostert, "Analysis and modeling of impulsive noise in broad-band powerline communications," IEEE Trans. Electromagn. Compat., vol. 44, pp. 249-258, Feb. 2002.

[2] O. Hooijen, "A channel model for the residential power circuit used as a digital communications medium," IEEE Trans. Electromagn. Compat., vol. 40, no. 4, pp. 331-336, 1998.

[3] M. G. Sanchez, L. de Haro, M. C. Ramon, A. Mansilla, C. M. Ortega, and D. Oliver, "Impulsive noise measurements and characterization in a UHF digital TV channel," IEEE Trans. Electromagn. Compat., vol. 41, no. 2, pp. 124-136, May 1999.

[4] D. Anastasiadou and T. Antonakopoulos, "Multipath characterization of indoor power-line networks," IEEE Trans. Power Del., vol. 20, no. 1, pp. 90-99, Jan. 2005.

[5] M. Zimmermann and K. Dostert, "A multipath model for the powerline channel," IEEE Trans. Commun., vol. 50, no. 4, pp. 553-559, 2002.

[6] H. Meng, Y. L. Guan, and S. Chen, "Modeling and analysis of noise effects on broadband power-line communications," IEEE Trans. Power Del., vol. 20, no. 2, pp. 630-637, Apr. 2005.

[7] P. Cuntic and A. Bazant, "Analysis of modulation methods for data communications over the low-voltage grid," in Proc. Int. Conf. Telecommun., vol. 2, Jun. 2003, pp. 643-648.

[8] Y. H. Ma, P. L. So, and E. Gunawan, "Performance analysis of OFDM system for broadband power line communications under impulsive noise and multipath effects," IEEE Trans. Power Del., vol. 20, no. 2, pp. 674-682, Apr. 2005 .

[9] D. Middleton, "Statistical-physical models of electromagnetic interference," IEEE Trans. Electromagn. Compat., vol. EMC-19, no. 3, pp. 106-127, Aug. 1977.

[10] _ "Canonical and quasi-canonical probability models of class-A interference," IEEE Trans. Electromagn. Compat., vol. EMC-25, pp. 76-106, May 1983.

[11] O. P. H. et al., "Detection and removal of clipping in multicarrier receivers,' Eur. patent Appl. EP1043874, Oct. 2000, Bull. 20000/41.

[12] N. P. Cowley, A. Payne, and M. Dawkins, "Cofdm."

[13] S. V. Zhidkov, "On the analysis of OFDM receiver with blanking nonlinearity in impulsive noise channels," in Proc. Int. Symp. Intell. Signal process. Commun. Syst., Nov. 2004, pp. 492-496.

[14] — "Analysis and comparison of several simple impulsive noise mitigation schemes for OFDM receivers," IEEE Trans. commun., vol. 56, no. 1, pp. 5-9, Jan. 2008.

[15] K. S. Vastola, "Threshold detection in narrow-band non-gaussian noise," IEEE Trans. Commun., vol. COM-32, no. 2, pp. 134-139, Feb. 1984.

[16] R. Ingram, "Performance of the locally optimum threshold receiver and several suboptimal nonlinear receivers for ELF noise," IEEE J Ocean. Eng., vol. OE-9, no. 3, pp. 202-208, Jul. 1984.

[17] S. V. Zhidkov, "Performance analysis and optimization of OFDM receiver with blanking nonlinearity in impulsive noise environment," IEEE Trans. Veh. Technol., vol. 55, no. 1, pp. 234-242, Jan. 2006.
[18] E. Alsusa and K. Rabie, "Dynamic peak-based threshold estimation method for mitigating impulsive noise in power-line communication systems," IEEE Trans. Power Del., vol. 28, no. 4, pp. 2201-2208, 2013.

[19] M. Ghosh, "Analysis of the effect of impulse noise on multicarrier and single carrier QAM systems," IEEE Trans. Commun., vol. 44, no. 2, pp. 145-147, Feb. 1996.

[20] D. Dardari, V. Tralli, and A. Vaccari, "A theoretical characterization of nonlinear distortion effects in OFDM systems," IEEE Trans. Commun., vol. 48 , no. 10 , pp. $1755-1764,2000$.

[21] R. van Nee and R. Prasad, "OFDM for wireless multimedia communications," Boston, MA, USA: Artech House, 2000.

[22] H. Ochiai and H. Imai, "On the distribution of the peak-to-average power ratio in OFDM signals," IEEE Trans. Commun., vol. 49, no. 2, pp. 282-289, Feb. 2001.

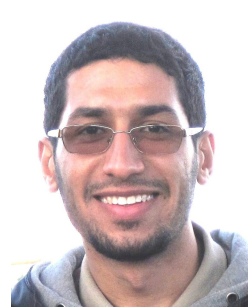

Khaled M. Rabie (S'12) received the B.Sc. degree in electrical and electronic engineering from the University of Tripoli, Tripoli, Libya, in 2008 and the M.Sc. degree (with Distinction) in communication engineering from the University of Manchester, Manchester, UK, in 2010. Since September 2011, he has been working toward the Ph.D. degree in the Microwave and Communications Systems (MACS) group of the University of Manchester. His current research interests are power-line communications including power-line channel modeling, interference and impulsive noise mitigation for smart-grid applications as well as multiple-input multiple-output (MIMO) systems.

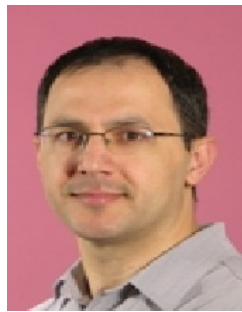

Emad Alsusa (M'06-SM'07) received a Ph.D. degree in electrical and electronic engineering from Bath University, Bath in 2000. Following his $\mathrm{PhD}$ he joined the School of Engineering and Electronics at Edinburgh University as a MobileVCE Postdoctoral Research Fellow, working on industrially led projects on link enhancement techniques for future high data rate wireless communication systems. In 2003, he joined the University of Manchester as an academic member of the school of Electrical and Electronic Engineering, where he lectures on communication engineering subjects. His research interests include signal processing and analysis of wireless communication networks, with particular focus on modulation and multiple access, channel estimation, coding, interference mitigation, multiuser detection, MIMO techniques and spectrum sensing techniques for cognitive radio applications. He served as a technical program committee member on numerous IEEE flagship conferences and chaired the Manchester EEE postgraduate conference in 2010 